\title{
Reduction of the Humidity Contained in the Harvest Cereals by the Means of in High Frequency Electromagnetic Field Processing
}

\author{
Cornelia Emilia Gordan $^{1}$, Ioan Mircea Gordan ${ }^{2}$, Vasile Darie Șoproni ${ }^{3}$ \\ Carmen Otilia Molnar ${ }^{4}$, Mircea Nicolae Arion ${ }^{5}$, Francisc Ioan Hathazi ${ }^{6}$ \\ Department of Electronics and Telecommunications, University of Oradea, România ${ }^{1}$ \\ Department of Electrical Engineering, University of Oradea, România ${ }^{2,3,4,5,6}$
}

\begin{abstract}
The new environmental friendly microwave technologies represents an important concern in the environmental policies. The use of microwave energy for the processing of different agricultural products presents the advantage of a green technology which allow a uniform distribution of electromagnetic and thermal field with a short relatively time of the process. In the paper is studied the microwave drying technology used in the drying process of oat seeds. In this sense the experiments were carried out for different working conditions of the equipment with respect to applied microwave power and obtained temperatures. A numerical model associated to the problem and solved by the means of finite element method is used. These allows us to obtain the electromagnetic field distribution through simulation inside the microwave dryer. The simulations were performed in order to obtain good quality products that may be used for seeding and food industry. The approached method is flexible so as it can be applied to all cereals.
\end{abstract}

Keywords-High frequency electromagnetic field; numerical simulation; microwave processing; oat drying; agricultural seeds

\section{INTRODUCTION}

In the context of the global economy, a major importance is the maintenance of the quality of stored agricultural products intended for use as seeding, the food industry and animal husbandry. Depending on weather conditions at harvest [1], the physical properties of agricultural seeds may be different, so when are harvested, they may have a different relative humidity having a maximum permissible value of $21 \%$. Ensuring the optimal storage conditions of agricultural products, in order to avoid their deterioration or contamination due to mycotoxin factors is required the ensuring a low moisture content of the samples. In this sense, in the specialized literature it is recommended that the maximum value of the humidity of the stored cereal agricultural products does not exceed the maximum value of $14 \%$. [2, 3] Consequently, regardless of the method, the moisture content of the harvested agricultural product must be reduced before storage.

Current trends in ensuring a healthier diet have stimulated the growing demand for oats for human consumption even though oats were initially used as feed. Farmers recognize the uniqueness of the nutritional qualities of oats, which have a high content in high quality protein with a low energy content, resulting in easier digestion.

Scientific research [2] in the field has shown that oat is a special cereal product, it does not contain gluten and has a high content of lipids beneficial to the body. In the case of oats, the enzymes associated with lipids are activated during industrial milling, so as oat oils are of nutritional importance in the content of oat products resulting from milling.

Among cereals, oats have the highest concentration of lipids that provide a significant amount of energy in human or animal nutrition, oat fatty essential oils are unsaturated with a high content of linoleic acid, an acid beneficial to the body and which allows the reduction of the amount of blood cholesterol serum [4]. Depending on the variety of oats [5], the concentration of lipids can vary and reaches values between 3.1-11.6\%, oils which can be easily extracted.

The variation of the individual concentration of fatty acids in oat crops depends on the type of selected oat and can be manipulated by genetic modification of the seeds. The medium values in composition of fatty acids from oat reaches the percentage of $0.6 \%$ for myritic acid, $18.9 \%$ for palmitic acid, $1.6 \%$ for stearic acid, $36.4 \%$ for the oleic acid and $40.5 \%$ for the linoleic acid [2].

The oat drying operation presumes reduction of its moisture content, through exposure to a thermal field of the sample. Thus, the reduction of the moisture content of the oats, without a qualitative deterioration of it, is a problem of interest, having a high degree of complexity. The reduction of the moisture content is achieved by exposing the sample to a thermal field [6, 7], which implies the continuous control of the temperature values of the thermal field so as the maximum value of the temperature during drying do not exceed 70 degrees.

The specialized literature [8-13] presents the versatility of adapting microwave technologies in different industrial applications. Unlike conventional drying methods [3], microwave drying technologies are environmentally friendly, having a number of advantages [8-13] such as selective heating by transmitting energy directly to the entire volume of the dielectric thus reducing processing time, and the energy consumed, ensuring a rapid release of moisture content. Also 
the process allows automatic real-time control of the entire process by continuously monitoring the humidity and temperature, the quality of the resulting product being superior compared to other drying methods. Compared to conventional installations, the microwave system has smaller construction dimensions, less components, respectively the maintenance costs are substantially reduced.

Researches in the field [14-16], point out the fact that an important issue is to determine the energy values that should be applied to the seed bed so as the free water to be removed without affecting the integrity of the sample. In the literature [17-19], are presented different computations approaches used for the complex analysis of the high frequency electromagnetic field problems. The researches performed in the paper study the microwave drying possibilities of the oats. For the beginning a numerical model associated to the problem is numerical computed by the means of finite element method, resulting the field values. Further, the obtained numerical results were used during experiments to set-up the microwave drying. The microwave drying process studied in the paper presents an important issue due to increase grain storing request once with the climate changing opening new directions for future researches in the field, due to its advantages in improving the drying process efficiency without affecting the quality of the products.

\section{THE DRYING MECHANISM}

The drying of agricultural cereal products is a simultaneous process of heat and mass transfer, in which the moisture content of the wet material (in this case oat seeds), is reduced to a level that gives it safety in storage and distribution, as well as in keeping its qualities. Researches in the field presents different technologies used for the reduction of relative humidity for different agricultural products [1], [4]. The drying operation involves in this case the transfer of heat to the macro-particles and can be performed by: convection, conduction, or high frequency electromagnetic radiation.

The convection drying is based on a convective heat transfer from the heated air to the solids (in our case oats) [1]. The hot air is forced to pass through a fixed layer of grains, heats them and then drives to the process of diffusion of moisture that results during drying. Although the drying rate can be increased by increasing the air temperature, there is still a limit to the air temperature around which the phenomenon of grain overheating can occur, especially in the areas adjacent to the air inlet. Overheating leads to cracking of the grains [4], decrease of the standard weight, and decrease of the content of trace elements and of course their germination capacity. Usually the convection drying process [2] is performed with a high energy consumption, because the entire seeds bed is heated to the evaporation temperature, to ensure an adequate heat flow. For this, powerful electric fans are used to blow hot air through the material, air that is heated using conventional fuels (gaseous or liquid), which by combustion pollutes the atmosphere.

The conduction drying [3], reduces moisture when the grains come in contact with a heated surface, or other moisture-absorbing granular material. Currently, the drying of agricultural cereal products by conduction using other granular materials (such as silica gel, or zeolites, etc.) is restricted due to the separation problems involved in the recycling process of macro-particles used as a medium for drying grains.

Currently, the trends are to develop new technologies that use microwaves and radio frequency that produce the inverse temperature gradient, so as the temperature is produced directly in the sample instead of at its surface [5], [7]. The energy absorption of the microwaves in the sample and the generation of the thermal field in its, is mainly due to the presence of water in free form.

The water gradient inside the sample can be estimate through its magnitude as follows:

$\mathrm{d}=\frac{2 \cdot\left(\mathrm{h}_{\mathrm{i}}-\mathrm{h}_{\mathrm{he}}\right)}{\mathrm{s}}$

where it was considered the moisture inside the sample $h_{i}$, the moisture of surrounding environment according to hygroscopic balance $h_{h e}$, and the thickness of the material.

The detailed mechanics [8], [10] of the conversion of microwave energy into heat, concludes that the dielectric parameters of the materials and the frequency are the main factors that lead to the heating of the materials.

The dielectric properties of a considerate oat sample exposed to microwave field is straight dependent on moisture contain, temperature and the field frequency. In the technical literature the dielectric properties of the oat sample are described through there material permittivity [6]. The complex permittivity can be written as function of dielectric constant $\varepsilon^{\prime}$, dielectric loss factor $\varepsilon^{\prime \prime}$ and the permittivity of free space $\varepsilon_{0}$ :

$\varepsilon^{*}=\left(\varepsilon^{\prime}-j \varepsilon^{\prime \prime}\right) \varepsilon_{0}$

where $j$ is the complex operator.

Fig. 1 and Fig. 2 presents the variation of dielectric constant and the loss factor function of frequency and moisture. As can be seen the dielectric properties of the sample are straight dependent on the field frequency, samples moisture, varying also with temperature.

It is known the fact that the dielectric properties of a sample also affect the power attenuation of the electromagnetic waves as they penetrate the loss material so as the thermal field distribution. The penetration depth $\left(\mathrm{p}_{\mathrm{d}}\right)$ can be defined as the distance from the surface of the sample at which the power drops to $1 / \mathrm{e}(36.8 \%)$ from its value at the surface of the sample:

$\mathrm{p}_{\mathrm{d}}=\frac{\lambda_{0} \sqrt{\varepsilon^{\prime}}}{2 \pi \varepsilon^{\prime \prime}}$

where $\lambda_{0}$ is the free space wavelength.

Prediction of the penetration depth during microwave processing with respect to the irregular dependence on dielectric loss factor with the temperature and the moisture content in the case of oat seeds, during microwave drying process is a tedious task [14]. 
The large versatility of microwave processing technology increases its request in different commercial domains $[5,10]$. The essential conditions in the case of microwave processing are represented by the precise control of the applied microwave power and the uniform distribution in the sample of the thermal [16], so the microwave applicator must be designed to meet these objectives. The applicator surface wave is one of those devices that have been used successfully along with coupling microwave energy transfer [13, 20].

During microwave drying of oat, a complicated diffusion problem appears. Thermal field dispersion in the seed bed represents an important issue due to the inner evaporation where the nonhomogeneous pressure field interferes due to vapours [12]. In this case, the water migrates from the high temperatures region to areas with lower temperatures, resulting the condensation in cold spots. The fast occurrence of vapours from the inside structure of the sample can cause its destruction. From these reasons the microwave drying process must be accomplished by air stream.

Starting from the above issues is studied the continuous microwave drying of oat seeds. The considered microwave drying system is designed with six $1 \mathrm{~kW}$ magnetrons and permit the continuous feeding and processing of the oat seeds. The oat seeds displacement inside the microwave applicator is realised with a helical screw conveyor, which ensure also the homogenization of the thermal field resulted in oats once with it exposer to microwave field. A schematic representation of the system is presented in Fig. 3.

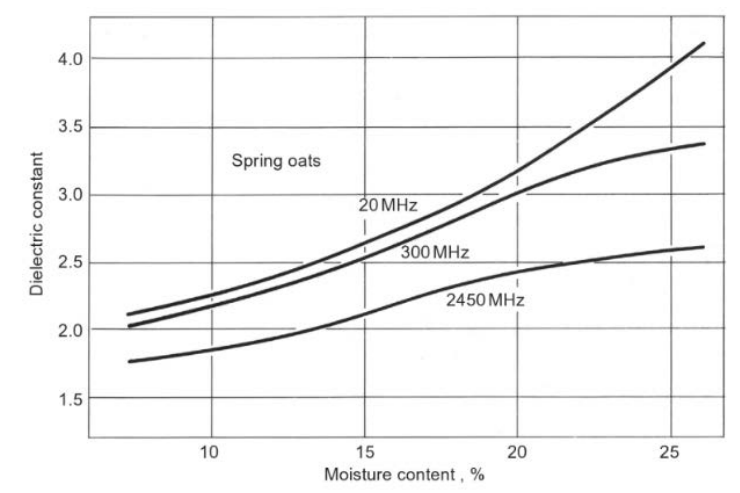

Fig. 1. Variation of the Dielectric Constant of Spring Oats with Moisture Contain at Indicated Frequency [6].

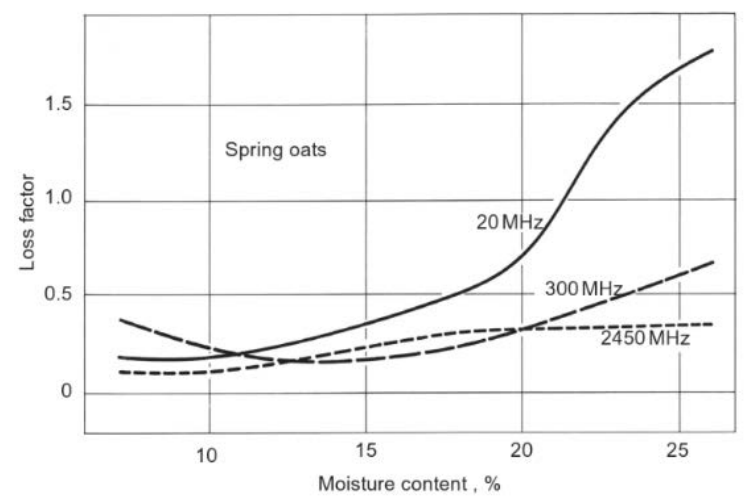

Fig. 2. Variation of the Loss Factor of Spring Oats with Moisture Contain at Indicated Frequency [6].

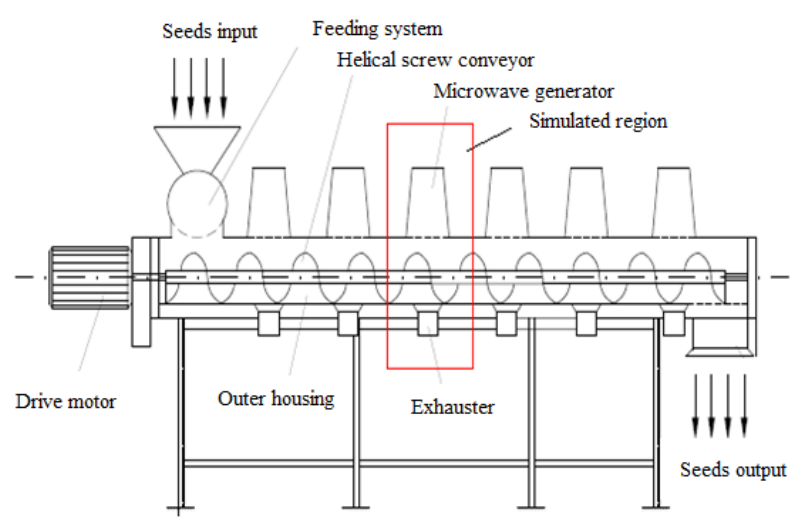

Fig. 3. Microwave Drying System.

\section{FIELD PROBLEM FORMULAE}

The design of microwave heating systems with respect to applicator sizing and the selection of the appropriate microwave generators is not an easy issue. Researches from the field offers analytical models for their design [17-19]. Approach takes into account the dielectric parameters modification during their microwave processing, becoming a complex problem. Sometimes from these reasons, the approach of the problem simplified [17].

The drying proposed procedure is initially numerical analysed in order to obtain an overview upon the field values inside the microwave applicator. The numerical simulation of the microwave drying process it was proceed by considering the frequency transient state. The obtained numerical results with respect to in time exposure of the sample to microwave field will be used in the second part of the research to setup the working conditions of the microwave drying system used for spring oat drying. The considered numerical model presumes to solve Maxwell's equations for the sinusoidal regime. In this sense it is supposed as known the transversal electric wave $E_{t}$ on port. On the rest of the conducting walls the transversal electric wave $E_{t}$, as well as the internal and external field sources will be imposed equal to zero. The considered model will presume to solve of the equation (4), which takes into account the relative magnetic permeability $\mu_{\mathrm{r}}$; the electric field strength $\boldsymbol{E}$; the wave factor k; the relative electric permittivity $\varepsilon_{\mathrm{r}}$; the electric conductivity $\sigma$; the pulsation of sinusoidal quantities $\omega$ and the free space permittivity $\varepsilon_{0}$ :

$$
\nabla \times\left(\mu_{\mathrm{r}}^{-1} \nabla \times \boldsymbol{E}\right)-\mathrm{k}^{2}\left(\varepsilon_{\mathrm{r}}-\mathrm{j} \sigma / \omega \varepsilon_{0}\right) \boldsymbol{E}=0
$$

In the model will be considered similar working conditions of the microwave drying system. In this sense the applicators work frequency is $2.45 \mathrm{GHz}$, as well as the cavity excitation mode, the dielectric's characteristics and the above boundary conditions.

Thermal field developed into processed sample in the microwave applicator is computed for the transient regime, which can be associated to Joule's effect [17], considering the heating of the material, and the heat transfer by conduction.

$$
-\operatorname{divk} \operatorname{grad} T+\mathrm{c} \frac{\partial T}{\partial t}=p
$$


where were considered: the volume power density $\mathrm{p}$ in $\left[\mathrm{W} / \mathrm{m}^{3}\right]$, the density $\rho\left[\mathrm{kg} / \mathrm{m}^{3}\right]$, the specific heat $\mathrm{C}_{\mathrm{p}}$ in $\left[\mathrm{J} / \mathrm{kg}^{\circ} \mathrm{C}\right]$, the temperature $\mathrm{T}\left[{ }^{\circ} \mathrm{C}\right]$, the thermal conductivity $\lambda$ $\left[\mathrm{W} / \mathrm{m}^{\circ} \mathrm{C}\right]$.

In order to solve the thermal field question, the following boundary condition is imposed:

$-\lambda \frac{\partial \mathrm{T}}{\partial \mathrm{n}}=\alpha\left(\mathrm{T}-\mathrm{T}_{0}\right)$

where was considered the thermal convection coefficient $\alpha$ in $\left[\mathrm{W} / \mathrm{m}^{2}{ }^{\circ} \mathrm{C}\right]$ and the temperature on the dielectric's boundary domain and in the air $\mathrm{T}_{0}$ in $\left[{ }^{\circ} \mathrm{C}\right]$.

The initial value of the temperature used for the dielectric's boundary in the air domain $\mathrm{T}=\mathrm{T}_{0}\left(\mathrm{~T}_{0}=23^{\circ} \mathrm{C}\right)$ was considered for the thermal problem in the dielectric's boundary condition. The thermal insulation is imposed as: $-\mathbf{n} \cdot(-\lambda \nabla \mathrm{T})=0$.

The whole domain is meshed by using tetrahedral elements, resulting the discretization network. Equation (4) is solved by the means of finite element method by using the same mesh as the electric problem, with an extra fine mesh in the processed sample and a less fine in the rest of the geometry.

Thermal field in the processed sample and its dispersion represents an important issue. Due to the inner evaporation the complicated water diffusion problem appear due to the nonhomogeneous pressure field interferes of the vapours. The fast occurrence of vapours from the inside structure of the sample can cause its destruction. Taking into account this concerns, the maximum temperature of the sample must be limited to maximum $70^{\circ} \mathrm{C}[2,4]$. In consequence will be considered only the evaporation of the surface of the sample, the inner evaporation being neglected. The evaporation speed over the sample surface is straight dependent of the surface sample temperature and the surrounding temperature with respect to the vapours saturation degree, air pressure and air flow in the vicinity of the sample [17].

It will be admitted that the evaporation speed on the surface unit will be the same on the entire surface with it linearly dependence on temperature:

$$
\frac{\mathrm{d} \tau_{\mathrm{s}}}{\partial t}=w\left(T-T_{e}\right)
$$

where the evaporated volume of water on the surface unit is $\tau_{\mathrm{s}}$, and evaporated volume of water at a difference of $1^{\circ} \mathrm{C}$ is $\mathrm{W}$.

If $\Lambda$ is the latent heat of vaporization volume, the heat loss due to vaporization on the surface will reduces the temperature on the surface in the manner of thermal convection. From this reason, we can consider the vaporization by introducing the fictive convection coefficient, according to the relation:

$\alpha_{\mathrm{ech}}=\alpha+\Lambda w$

This coefficient will be considered as part of boundary condition, so we have:

$-\lambda \frac{\partial T}{\partial n}=\alpha_{\mathrm{ech}}\left(T-T_{e}\right)$

To determine the temperature field over the interval $\left[\mathrm{t}_{\mathrm{i}}\right.$, $t_{i+1}$ ] will allow the determination of water volume evaporated over this interval, according to relation (7):

$$
-\left(V_{\text {water }}^{i+1}-V_{\text {water }}^{i}\right)=\int_{t_{i}}^{t_{i+1}}\left(\oint_{\partial \Omega} w T \mathrm{~d} l\right) \mathrm{d} t
$$

The moisture becomes:

$$
\mathrm{m}^{i+1}=\frac{\gamma_{\text {water }} V_{\text {water }}^{i+1}}{\gamma_{\text {water }} V_{\text {water }}^{i+1}+\gamma_{s} V_{s}}
$$

where: $V_{s}$ is the volume of the charge and $\gamma_{\text {water }}$ and $\gamma_{s}$, are water densities over the surface.

To determine the percentage of moisture removed from the seeds bed using weight of the sample before drying $m_{i}$ and after drying $\mathrm{m}_{\mathrm{u}}$ (STAS 10349 /1-87):

$$
H=\frac{\mathrm{m}^{i}-\mathrm{m}^{i+1}}{\mathrm{~m}^{i+1}} \times 100[\%]
$$

To the above mentioned conditions is added also the limitation condition of moisture leap: $\mathrm{m}^{i}-\mathrm{m}^{\mathrm{i}+1}<\mathrm{H}$, where $\mathrm{H}$ is the imposed moisture variation. These has to be smaller than the final moisture in order to avoid the apparition of the negative moistures. If the difference of moisture will exceed this limit, then the time step will get smaller. The material parameters of the sample depend on temperature and moisture, and are rectified for each time step.

\section{NUMERICAL SIMULATION AND RESULTS}

Numerical simulation was pursued to obtain the electromagnetic field dispersion in the dielectric's volume; temperature dependence on applied microwave power, and not least the drying characteristic of the considerate dielectric material (spring oat).

The numerical simulation is performed by using a numerical analysis software where a Frequency - Transient problem is defined. Due to the symmetry of the system was considered only the applicator associated to one of the magnetrons. The geometrical dimensions of the microwave applicator are defined as $345 \times 240 \times 250[\mathrm{~mm}]$, similar to the one of the laboratory equipment. The walls of the microwave applicator and waveguide are defined as Aluminium. The oat sample is placed inside the applicator with a $60[\mathrm{~mm}]$ air layer on top of it. During numerical computation, the spring oat with it properties for $22 \%$ humidity $\left(\varepsilon^{\prime}=2.5, \quad \varepsilon^{\prime \prime}=0.3\right.$ and $\operatorname{tg} \delta=0.14, \rho=780 \mathrm{~kg} / \mathrm{m}^{3}, \lambda=0.15 \mathrm{~W} / \mathrm{mK}$ and $C_{p}=1.5 \mathrm{~kJ} / \mathrm{kgK}$ were considered $[2,6]$. In the defined problem was considered an extra fine mesh for the dielectric material, and a coarse mesh for the rest of the analysed geometry.

In the following some results of the simulation are presented in order to distinguishing the details concerning 
electric field distribution in the seed bed placed into the applicator. For a better view in Fig. 4 is presented the complex electric field strength dispersion inside the microwave applicator and dielectric material is presented, pointing out the energy supplied through the microwave port, placed on the top of the microwave applicator.

The reflected power to the magnetron expressed through the S Parameters (dB) presented in Fig. 5 is computed also. Considering the obtained results, after the rigueur transformations, it observed that if the reflected power to the waveguide tends to minimum, a higher quantity of possible power may be absorbed by the dielectric material. Based on the obtained field values, it was computed the time dependence temperature in the dielectric material with respect to microwave power. The time dependence of temperature was computed in the volume of the sample. During computation was considered that the sample was exposed to constant microwave power for 300 seconds.

Fig. 6 presents the time variation of temperature for different vales of the applied microwave power. The considerate values used during computation are: in the first case was considered a 0.25 [W/g] applied microwave power; in the second case $0.50[\mathrm{~W} / \mathrm{g}]$; in the third case $0.75[\mathrm{~W} / \mathrm{g}]$; in the fourth case 1.00 [W/g]; and in the fifth case 1.25 [W/g]. Taking into account the fact that this drying process should be continuous, the exposure to microwave energy should be shorter to insure the processing of a larger amount of seeds, but without affecting their quality. In order to assure a good balance between the humidity release and the quality of the sample should not exceed $90^{\circ} \mathrm{C}$.

By analysing Fig. 6, we can see that for the first 2 cases, when the samples were exposed to $0.25[\mathrm{~W} / \mathrm{g}]$ and 0.50 [W/g] microwave power, the obtained temperatures in the seed volume can hardly reach $43.23\left[{ }^{\circ} \mathrm{C}\right]$ in the first case and $52.31\left[{ }^{\circ} \mathrm{C}\right]$ in the second case of analysis. By analysing the rest of the cases, when the sample was expose to $0.75[\mathrm{~W} / \mathrm{g}]$, $1.00[\mathrm{~W} / \mathrm{g}]$ and $1.25[\mathrm{~W} / \mathrm{g}]$ microwave power, the temperature reaches values of $67.14\left[{ }^{\circ} \mathrm{C}\right], 85.09\left[{ }^{\circ} \mathrm{C}\right]$ and $101.57\left[{ }^{\circ} \mathrm{C}\right]$.

The aim of research was to determine the most appropriate value of microwave power and processing time for the considerate microwave drying system in order to dry a considerate product without affecting its structure. From the specialty literature it is known that a temperature over $90^{\circ} \mathrm{C}$ can destroy the quality of agricultural products.

The most suitable value imposed for the microwave power with respect to the quality of the product for the 300 seconds exposure time should be with a value between $0,75-1,00$ [W/g]. In Fig. 7 is represented the drying characteristic for the 1,00 [W/g] imposed microwave power.

For each numerical simulation were recorded the temperatures in the whole volume of the dielectric material. The objective of the study is to find out the appropriate values for exposure time and the applied microwave power.

The most appropriate value of the microwave power and processing time in order to dry the mass of seeds without affecting its structure.

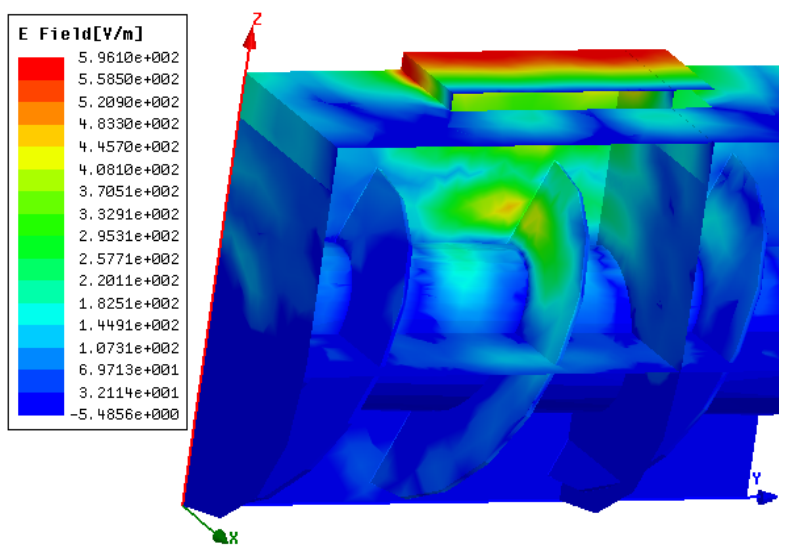

Fig. 4. Electric Field Strength Dispersion within the Microwave Dryer.

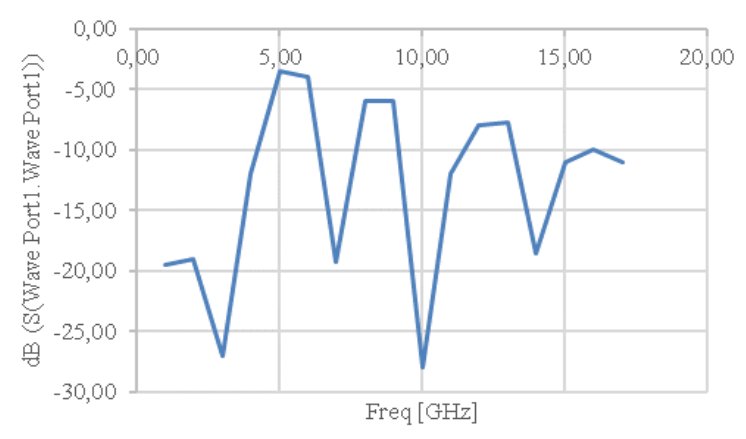

Fig. 5. Reflected Power to the Magnetron S Parameter.

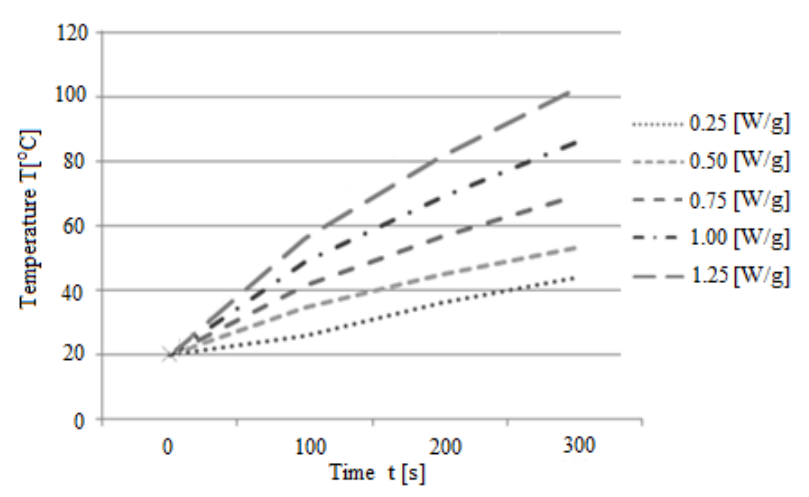

Fig. 6. Values of the Maximum Temperature Calculated in the Mass of the Oat According to Microwave Power at a Processing Time of 300[s].

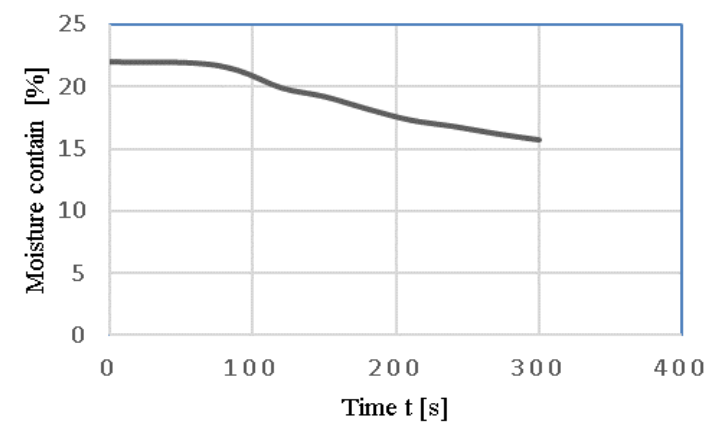

Fig. 7. In Time Moisture Dependence of the Oat Sample for of 1.00 [W/g] and Processing Time of 300 [s]. 


\section{CONCLUSIONS}

Unlike other methods of humidity reduction, the studied method has a higher value to allow a faster release of moisture content from the entire volume of material processed in the high frequency electromagnetic field, thus resulting in a substantial reduction in use time and thus creating efficiency technological process.

The obtained results present information regarding the improvement in quality of the stored oat seeds through an environmentally friendly technology.

The obtained numerical results offer important information regarding microwave drying procedure applied to oat seeds. Through simulation are known the characteristic parameters of the electromagnetic and thermal field drying process, values that are vital to adapt the operation parameters of the microwave drying systems.

In order to homogenize the thermal field inside the microwave applicator, it is necessary to set the grain in motion so as the drying process is uniform into entire volume of the product. Also, in order to facilitate the release of water vapours resulted on the seeds surface following the pressure field, the microwave drying equipment must be equipped with an exhaust system that removes excess water from the air through the air stream created in the cereal bed.

The increase of the microwave power to which the material to be processed is exposed, leads to a faster increase of the thermal field in the material. However, the maximum permissible grain temperature limit must be taken into account so that the quality of the product is maintained at high values, so as the applied microwave power must be adapted with respect to the in real-time temperature.

The aim of the study was to determine what conditions of temperature, humidity and power are favourable the percentage of moisture removed from the seeds (STAS 10349/1-87) bed. By analysing all results, we can say that the drying procedure which involve the use of constant applied microwave power of 1.00 [W/g] combined with air stream offers the fastest drying condition with respect to seeds quality.

\section{ACKNOWLEDGMENT}

This work was co-financed from the European Regional Development Fund through Sectoral Operational Program "Increase of the Economic Competitiveness" - POS - CCE 2007 - 2013, project number POS - CCE 1843 / 48800, "Increasing the capacity of research - development of the interdisciplinary laboratories for the technologies in electrical engineering”, developed by University of Oradea.

\section{REFERENCES}

[1] E.R. Carvalho, V.M. Francischini, Suemar Al. G. Avelar , J. Camargos da Costa, "Temperatures and periods of drying delay and quality of corn seeds harvested on the ears”, Journal of Seed Science, 41, 3, pp.336-343, Brazil, 2019.
[2] NIIR Board of Consultants \& Engineerss, "Handbook on Drying, Milling and Production of Cereal Foods: (Wheat, Rice, Corn, Oat, Barley and Sorghum Processing Technology) 2nd Revised Edition”, ASIA PACIFIC BUSINESS PRESS Inc., Oct 9, 2017.

[3] M. Zhang, B. Bhandari, Z. Fang, Handbook of Drying of Vegetables and Vegetable Products, CRC Press, 2017, USA.

[4] M.C. Elias, L.J. Marini, L. da C. Oliveira, E. Aosani, R.B. Prestes, L.C. Gutkoski, "Effects of air temperature in drying on white oat grains quality”, Proceedings of the 9th International Working Conference on Stored Product Protection, Campinas, São Paulo, Brazil, 2006.

[5] Kok Yeow You, "Emerging Microwave Technologies in Industrial, Agricultural, Medical and Food Processing”, BoD - Books on Demand, Jul 4, 2018 - Technology \& Engineering.

[6] S.O. Nelson, "Dielectric Properties of Agricultural Materials and their Applications”, Academic Press, 2015.

[7] F.I. Hathazi, V.D. Soproni, M.N. Arion, C.O. Molnar, S, Vicas, O. Mintas, "The Use of Microwave Drying Process to the Granular Materials”, (IJACSA) International Journal of Advanced Computer Science and Applications, 10, 12, 2019.

[8] M. Regier K. Knoerzer, H. Schubert "The Microwave Processing of Foods 2nd Edition”, Woodhead Publishing, 2016.

[9] A.C. Metaxas, R.J. Meredith, "Industrial Microwave Heating”, The Institution of Engineering and Technology, 2011, UK.

[10] E.T. Thostenson and T.W. Chou, "Microwave processing: fundamentals and applications”, Compos. Pt. A-Appl. Sci. Manuf., 30, pp. 1055-1071, 1999.

[11] G. Brodie, V. Mohan, P. Farrell, "Microwave and Radio-Frequency Technologies in Agriculture”, De Gruyter Open LTD, 2016, Germany.

[12] Wojdyło, A., Figiel, A., Lech, K., Nowicka, P., \& Oszmiański, J., "Effect of Convective and Vacuum-Microwave Drying on the Bioactive Compounds, Color, and Antioxidant Capacity of Sour Cherries”, Food and Bioprocess Technology, 7, 3, pp. 829-841, 2014.

[13] A.W. Kraszevvski and S.O. Nelson, Microwave Techniques in Agriculture, Journal of Microwave Power and Electromagnetic Energy , Volume 38, 2003 - Issue 1, pp 13-35, 2016.

[14] D. U. Yu,B. L. Shrestha \&O. D. Baik, "Radio Frequency Dielectric Properties of Bulk Canola Seeds under Different Temperatures, Moisture Contents, and Frequencies for Feasibility of Radio Frequency Disinfestation”, International Journal of Food Properties , 18, 12, 27462763, 2015.

[15] S. Mailadil, R. Ubic, H. Jantunen, "Microwave Materials and Applications”, John Wiley \& Sons, Ltd, 2017.

[16] L. Bandici, T. Leuca, S Coman, "The use of microwave field energy in the drying process of wooden sticks”, 14th International Conference on Engineering of Modern Electric Systems (EMES), IEEE, Romania, 2017.

[17] T. Leuca, A. Palade, I. Hănțilă, L. Bandici, "The use of hybrid finite element - boundary element method for all the analysis of the operating parameters of a radio-frequency dryin instalation”, Revue roumaine des sciences techniques, Série Électrotechnique et Énergétique, 56, 4, pp. 367-376, 2011.

[18] H. Narang, F. Wu, A. Ogunniyan, "Numerical Solutions of Heat and Mass Transfer with the First Kind Boundary and Initial Conditions in Capillary Porous Cylinder Using Programmable Graphics Hardware”, (IJACSA) International Journal of Advanced Computer Science and Applications, 7, 6, 2016.

[19] Hemis, M., Choudhary, R., \& Watson, D. G., “A coupled mathematical model for simultaneous microwave and convective drying of wheat seeds”, Biosystems Engineering, 112, 3, pp. 202-209, 2012.

[20] Cusacani Guerrero, Julio Agapito, Roman-Gonzalez Avid, “Antennas of Circular Waveguides”, (IJACSA) International Journal of Advanced Computer Science and Applications, 10, 8, 2019. 